\title{
Ameloblastic fibro-odontosarcoma of the mandible with active epithelial proliferation: A rare case report
}

\author{
HAOMAN NIU ${ }^{1}$, JUNYU LIU ${ }^{1}$, YU CHEN ${ }^{2}$ and NING GENG ${ }^{2}$ \\ ${ }^{1}$ State Key Laboratory of Oral Disease and ${ }^{2}$ Department of Oral Pathology, West China Hospital \\ of Stomatology, Sichuan University, Chengdu, Sichuan 610041, P.R. China
}

Received November 3, 2016; Accepted May 3, 2017

DOI: $10.3892 / \mathrm{mco} .2017 .1448$

\begin{abstract}
Ameloblastic fibro-odontosarcoma (AFOS) is an extremely rare subtype of odontogenic sarcoma, with no more than 19 cases reported in the English literature to date. AFOS is a biphasic neoplasm, with deposits of dentin and enamel matrix. We herein present a case of AFOS with active epithelial proliferation in a 31-year-old female patient. The patient was referred to the West China Hospital of Stomatology (Chengdu, China) due to a 6-month history of a swelling in the left mandible. Following clinical and radiological examination, the initial preoperative diagnosis was ameloblastoma, with local invasion and the possibility of malignant transformation. Left hemimandibular resection was subsequently performed. The postoperative histopathological diagnosis was AFOS, accompanied by active epithelial proliferation. Immunohistochemically, cytokeratin (CK)14 and CK19 were intensely positive in the epithelium, whereas the mesenchymal cells were strongly positive for vimentin. The Ki-67 labeling index was considerably higher in the mesenchymal component (mean, 40\%) compared with that in the epithelial element (mean, 5-8\%). Three months after the surgical procedure, the patient remained clinically and radiologically disease-free.
\end{abstract}

\section{Introduction}

Malignant odontogenic neoplasms account for a small percentage of all odontogenic tumors and may be classified as carcinomas, sarcomas or carcinosarcomas (1). Ameloblastic fibro-odontosarcoma (AFOS) is an extremely rare odontogenic sarcoma, composed of benign odontogenic epithelium, a malignant mesenchymal component, and dental hard tissue (1). AFOS may either arise as a de novo lesion of the jaws $(2,3)$, or following the malignant transformation of a pre-existing ameloblastic fibro-odontoma (AFO) (4-6). AFOS usually

Correspondence to: Mr. Ning Geng, Department of Oral Pathology, West China Hospital of Stomatology, Sichuan University, No. 14, Section 3, Renminnan Road, Chengdu, Sichuan 610041, P.R. China E-mail: gengning@scu.edu.cn

Key words: ameloblastic fibro-odontosarcoma, odontogenic tumor, odontogenic sarcoma, histopathological, immunohistochemistry occurs in the second and third decades of life, with a predilection for the mandible (2-6). Due to its rarity and the lack of clinicopathological information, the diagnosis of AFOS is challenging. We herein report a de novo case of AFOS of the left mandible in a 31-year-old female patient, exhibiting active epithelial proliferation, which is considered as an uncommon finding in AFOS. In addition, the clinicopathological characteristics, clinical management and prognosis were also discussed, combined with a review of the relevant literature.

\section{Case report}

In September 2006, a 31-year-old woman was referred to the West China Hospital of Stomatology (Chengdu, China) due to a 6-month history of a swelling in the left mandible. The patient complained that the mass had expanded over the last 2 months, with associated pain.

On extraoral evaluation, the patient's face was asymmetrical due to a sizeable swelling over the left mandible, accompanied by limitation in opening the mouth. Intraorally, an exophytic neoplasm was observed, extending from the left lower premolar to the ascending ramus of the left mandible, measuring $\sim 8 \times 6 \times 4 \mathrm{~cm}^{3}$, with migration of teeth $43-44$ and missing teeth 45-47. No evidence of regional lymph node or distant metastasis was detected. The panoramic radiographic examination revealed a sizeable multilocular radiolucent lesion in the left mandible. The neoplasm extended from the left canine to the ramus of the left mandible, with indistinct margins and local perforation of the cortical plate. In addition, irregular radiopaque foci were observed within the lesion (Fig. 1).

The initial preoperative diagnosis was ameloblastoma, with local invasion and the possibility of malignant transformation. A left hemimandibular resection was performed, with tumor-free margins. Grossly, the tumor was exophytic and fleshy, with considerable destruction and replacement of the left mandible. The cut surface was grayish brown, with scattered firm tissue and hemorrhagic areas (Fig. 2).

Microscopic examination of the hematoxylin and eosin-stained slides showed a biphasic tumor with two major components, namely the benign odontogenic epithelial islands and the surrounding anaplastic mesenchymal component, with hyalinized areas at the epithelial-mesenchymal interface (Fig. 3A). The structure of the epithelial islands resembled that 
of the enamel organ, with a central area simulating the stellate reticulum cells outlined by a single layer of cuboidal or columnar ameloblast-like cells arrayed in a palisading pattern (Fig. 3B). The majority of the epithelial component displayed benign characteristics, but a few epithelial cells proliferated actively, with hyperchromatic cytoblasts and mitotic figures [mean, 2-4 mitoses/10 high-power fields (HPFs)] (Fig. 3C). The mesenchymal component exhibited cytological characteristics typical of malignancy, with spindle-to-ovoid shaped cells arranged in a fascicular pattern. The cells exhibited obvious nuclear pleomorphism and active mitoses (mean, 20 mitoses/10 HPFs) (Fig. 3D). In addition, irregular dysplastic dentin and enamel matrix were identified adjacent to the epithelial structures (Fig. 3E and F).

Immunohistochemical (IHC) staining was performed for cytokeratin (CK)14 and CK19, vimentin and Ki-67. Intense membranous and cytoplasmic staining for CK14 and CK19 were observed in the epithelium, with negative expression in the mesenchymal background (Fig. 4A and B). The mesenchymal cells were strongly positive for vimentin, which was negative in the epithelial component (Fig. 4C). The Ki-67 labeling index was considerably higher in the mesenchymal component (mean, 40\%) compared with the epithelial component (mean, 5-8\%) (Fig. 4D). One ameloblastoma (AB) and one ameloblastic fibroma (AF) case with the same markers were used for comparison, and were found to share the same staining pattern as AFOS regarding CK14, CK19 and vimentin; however, the Ki-67 labeling index in those two cases was very low compared with AFOS (mean, $3 \%$ in $\mathrm{AB}$ and $<2 \%$ in $\mathrm{AF}$ ) (Fig. 4E and F; Table I).

Finally, combined with the abovementioned examinations, a final diagnosis of AFOS was made. At 3 months postoperatively, clinical and radiographic follow-up of the patient revealed no recurrence or indication of metastasis. The patient was then lost to follow-up.

\section{Discussion}

AFOS is an extremely rare subtype of odontogenic sarcoma. The 2005 WHO classification (1) divided odontogenic sarcomas into two categories according to the presence of dentin and enamel matrix (without clinical or prognostic significance) as follows: Ameloblastic fibrosarcoma (AFS) and ameloblastic fibro-odontosarcoma/fibrodentino-sarcoma (AFOS/AFDS). The relative information is sparse, with no more than 73 cases $(7,8)$ of AFS and 19 cases of AFOS/AFDS (8 females) (2-6) reported in the English literature to date. In addition, AFOS has also been reported in a bovine mandible (9). Due to its rarity, the diagnosis of AFOS may be challenging, and it mainly relies on clinicopathological findings and radiological examination.

Clinically, a painful swelling is the most common complaint, with a reported duration of symptoms from 2 months (2) to 12 years (10). AFOS occurs at a mean age of 23.5 years (3), with a wide age range of $4-83$ years $(2,3)$. The male:female ratio is $1.2: 1$ according to the cases published to date (2-6). The majority of the cases involve the mandible, with a predilection for the retromolar region and the mandibular ramus, with only one reported case located in the maxilla (6). AFOS may either arise as a de novo lesion $(2,3)$ or from the

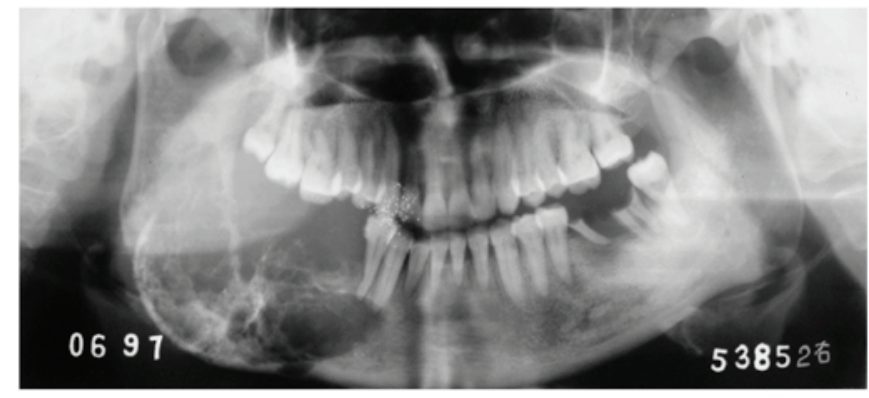

Figure 1. Panoramic radiograph showing an ill-defined multilocular radiolucent lesion extending from the left canine to the ramus of the left mandible, with local perforation of the cortical plate and irregular radiopaque foci within the entity.
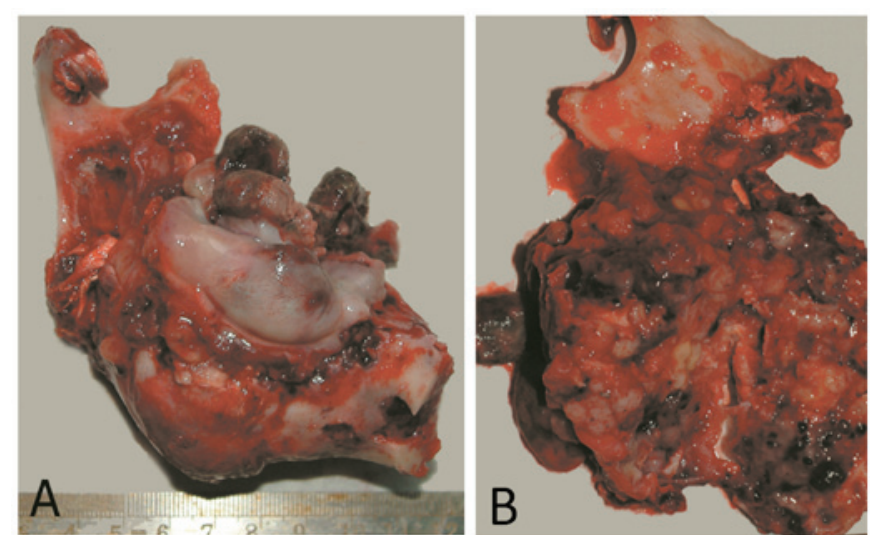

Figure 2. (A) Gross view and (B) cut surface of the neoplasm showing an exophytic, fleshy mass within the mandible, with a grayish brown color, along with scattered firm tissue and hemorrhagic areas.

malignant transformation of a pre-existing AFO (4-6). The mechanism underlying this conversion has not been fully elucidated. Radiographically, AFOS usually presents as a unior multilocular expansile radiolucent lesion, with ill-defined borders and focal dense opacities. When a tumor exhibits a poorly circumscribed outline and perforation of the cortex, the possibility of a malignant odontogenic tumor should be taken into consideration (2-6).

The case presented herein occurred in a 31-year-old female in the left mandible as a de novo lesion with a history of 6 months. The clinical and radiological examination suggested the possibility of malignancy and the patient underwent hemimandibular resection. Based on the abovementioned assessments alone, the risk of a false diagnosis is high. However, preoperative incisional biopsy and intraoperative frozen section biopsy may improve the accuracy of the diagnosis, preventing surgical overtreatment. The definitive diagnosis of AFOS is based on the postoperative histopathological examination.

Microscopically, this lesion exhibits the characteristic architecture of benign epithelial islands/cords enmeshed in the malignant mesenchymal component, with focal deposits of dentin and enamel matrix. The odontogenic epithelium elements are composed of columnar or cuboidal cells at the periphery arranged in a palisading pattern, exhibiting reverse polarization of the nuclei away from the basal membrane. The central areas of the epithelial islands/cords are less cellular, 
Table I. Comparison of immunohistochemical staining characteristics of AFOS, AB and AF.

\begin{tabular}{|c|c|c|c|c|c|c|}
\hline \multirow[b]{2}{*}{ Staining } & \multicolumn{2}{|c|}{ AFOS } & \multicolumn{2}{|c|}{$\mathrm{AB}$} & \multicolumn{2}{|c|}{$\mathrm{AF}$} \\
\hline & Epithelial & Mesenchymal & Epithelial & Mesenchymal & Epithelial & Mesenchymal \\
\hline CK14/19 & + & - & + & - & + & - \\
\hline Vimentin & - & + & - & + & - & + \\
\hline Ki67 & $5-8 \%$ & $40 \%$ & $3 \%$ & - & $<2 \%$ & $<2 \%$ \\
\hline
\end{tabular}

AFOS, ameloblastic fibro-odontosarcoma; AB, ameloblastoma; AF, ameloblastic fibroma; CK, cytokeratin.
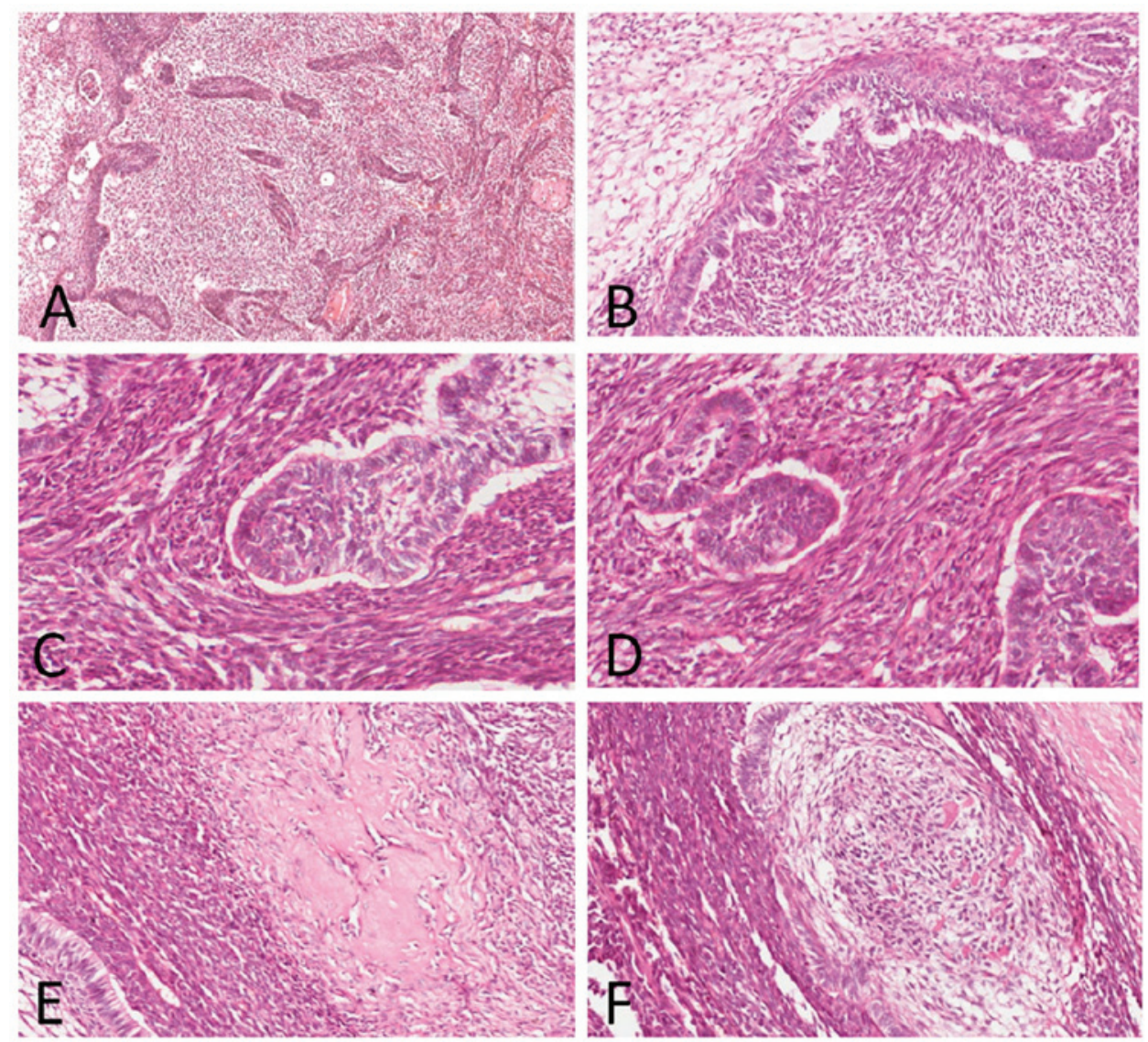

Figure 3. Histopathological findings of ameloblastic fibro-odontosarcoma. (A) The tumor was biphasic, with two major components: Islands/cords of benign odontogenic epithelial cells and anaplastic mesenchymal cells [hematoxylin and eosin (H\&E) staining; magnification, x40]. (B) A single layer of palisading cuboidal or columnar ameloblast-like cells were observed, surrounding a central area simulating stellate reticulum cells, resembling the structure of the enamel organ (H\&E staining; magnification, x100). (C) Epithelial cells exhibiting hyperchromatic nuclei with coarse chromatin, irregular nuclear margins and mitotic figures (H\&E staining; magnification, $\mathrm{x} 400$ ). (D) The mesenchymal background presented typical cytological characteristics of malignancy (H\&E staining; magnification, $\mathrm{x} 400$ ). (E and F) Deposits of irregular dysplastic dentin material and enamel matrix in the tumor adjacent to the epithelial structures. (H\&E staining; magnification, $\mathrm{x} 400)$.

edematous, mimicking the stellate reticulum of the enamel organ. The mesenchymal component includes spindle-to-ovoid cells exhibiting the characteristics of malignancy, including cell pleomorphism, nuclear atypia and hyperchromatism, with numerous mitotic figures. Occasionally, bizarre giant cells may be identified (2-6). In addition, IHC examination is a useful method for accurate diagnosis and prognosis evaluation. Vimentin is strongly positive in the mesenchymal component, and intense staining of CK14, CK19 and pan-CK may be detected in the epithelium. The malignant mesenchymal component highly expresses proliferating cell nuclear antigen and $\mathrm{Ki}-67$, which are negative or poorly expressed in the epithelial element (2-6).

In the present case, part of the odontogenic epithelium exhibited active proliferation, accompanied by deposits of immature dentinoid and enameloid matrix. The same appearance may be observed in AFO, but not in AB. Thus, we hypothesized that the odontogenic epithelium may induce the formation of dentin and enamel matrix under the influence of the benign or malignant mesenchymal component in biphasic odontogenic tumors. This may be an important finding in subsequent experiments on the induction of dental hard tissue. 

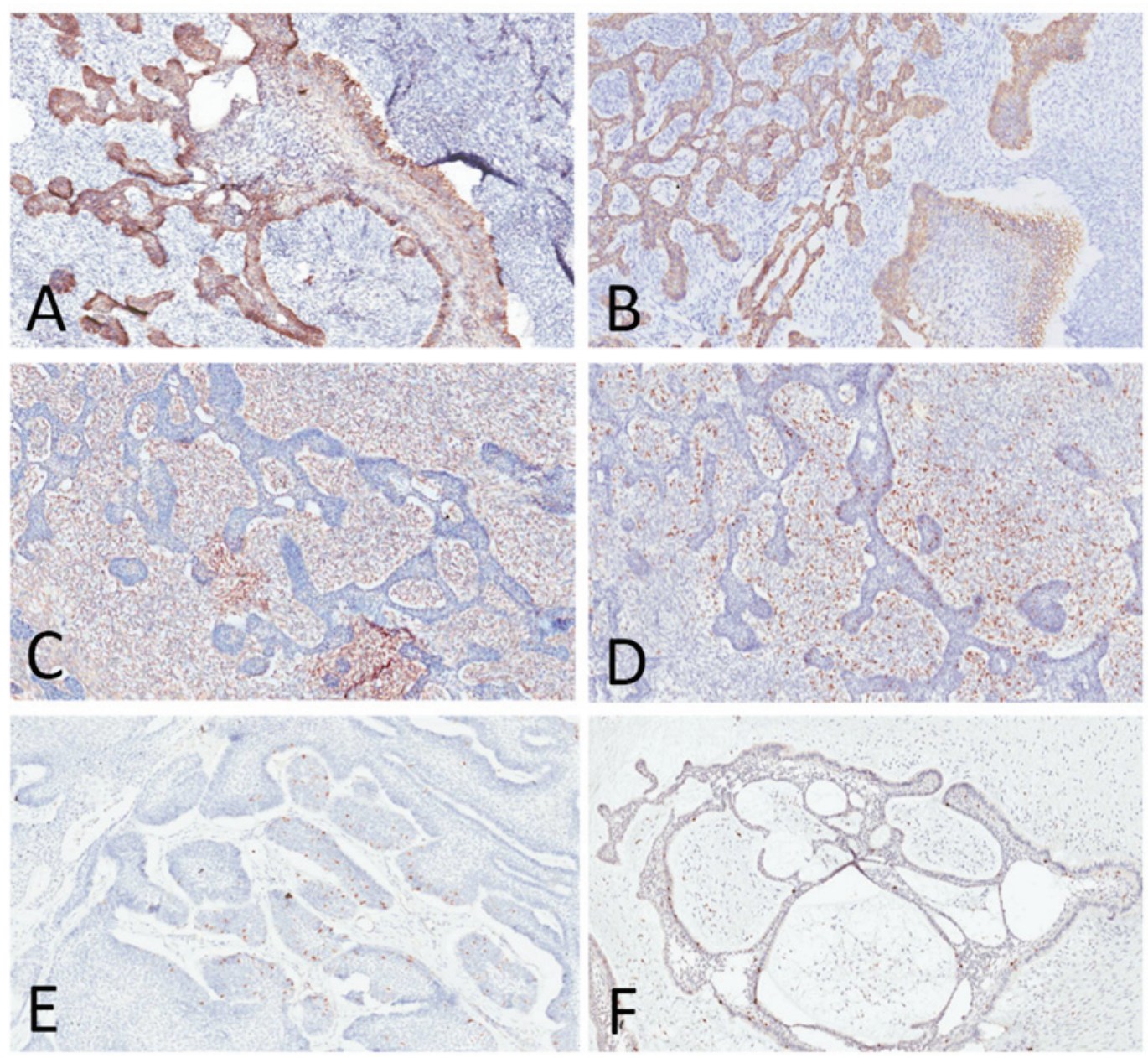

Figure 4. Immunohistochemical staining of ameloblastic fibro-odontosarcoma (AFOS), ameloblastoma (AB) and ameloblastic fibroma (AF) (A and B) Cytokeratin (CK)14 and CK19 staining in AFOS was positive in the epithelial component and negative in the mesenchymal background (magnification, $\mathrm{x} 40$ ). (C) Vimentin staining in AFOS was positive in the mesenchymal component and negative in the epithelium (magnification, $\mathrm{x} 40$ ). (D) The Ki-67 labeling index in AFOS was higher in the mesenchymal component (mean, 40\%) compared with the epithelial component (mean, 5-8\%) (magnification, $\mathrm{x} 40$ ). (E and F) The Ki-67 labeling index in AB and AF was lower compared with AFOS (mean, 3\% in AB and <2\% in AF) (magnification, $\mathrm{x} 40$ ).

AFO, a benign biphasic neoplasm with the same epithelial characteristics and hard dental tissue, is the main differential diagnosis in AFOS. Unlike AFOS, the mesenchymal component of AFO exhibits a benign appearance. The IHC staining of $\mathrm{Ki}-67$ is of great significance in distinguishing AFO from AFOS. As approximately one-third of AFOS cases are transformed from a pre-existing AFO, serial extensive sampling of the resected AFO specimen is crucial for elucidating the possible stepwise progression of AFO to AFOS. In addition, AFOS should be differentiated from ameloblastic carcinosarcoma, which is a biphasic malignant tumor with both epithelial and mesenchymal malignant components (2-6).

AFOS is usually considered as a low-to-intermediate-grade malignant tumor, with locally aggressive behavior and a recurrence rate of $10 \%$. Two cases with recurrence $(6,11)$ have been reported, one of which involved the base of the skull (11). By contrast, regional lymph node and distant metastases are rare. Only 1 of 20 cases developed local metastasis (10).

Due to the rarity of AFOS and the overall lack of experience with the treatment of this lesion, a standard of treatment has not been established. To date, radical resection with clear margins is the preferred treatment strategy. Long-term follow-up is crucial. Adjuvant radiotherapy and chemotherapy have been successfully used in some odontogenic sarcoma cases $(12,13)$. Chemotherapy with ifosfamide and doxorubicin and corresponding consolidated treatment by re-irradiation has been recommended for AFOS by Gatz et al (6). However, lung metastases were found in a patient 20 months after surgery and postoperative chemotherapy (14). Therefore, due to its uncertain benefits, the use of adjuvant radiochemotherapy remains a subject of debate.

As AFOS is a rare subtype of odontogenic sarcoma, its diagnosis and treatment may be challenging. Clinical assessment and X-ray examination play an important role, but the definitive diagnosis is based on histopathological examination. Radical resection is currently considered the optimal treatment and long-term follow-up is crucial. The aim of the present case report was to provide detailed information in order to enrich the database and improve our understanding of this rare tumor.

\section{Acknowledgements}

The present study was supported by the Chengdu Huimin Project of Science and Technology (2015-HM01-00412-SF). 


\section{References}

1. Barnes L, Eveson J, Reichart P and Sidransky D: World Health Organization classification of tumors. Pathology and genetics of head and neck tumors. Lyon: IARC Press: 294-295, 2005.

2. Wang S, Shi H, Wang P and Yu Q: Ameloblastic fibro-odontosarcoma of the mandible: Imaging findings. Dentomaxillofac Radiol 40: 324-327, 2011

3. Chen SJ, Zheng XW, Lin X and Liu H: Ameloblastic fibro-odontosarcoma of the mandible in a pediatric patient. Eur Ann Otorhinolaryngol Head Neck Dis 133: 419-421, 2016.

4. Mainenti P, Oliveira GS, Valério JB, Daroda LS, Daroda RF, Brandão G and Rosa LE: Ameloblastic fibro-odontosarcoma: A case report. Int J Oral Maxillofac Surg 38: 289-292, 2009.

5. Reiser V, Alterman M, Shuster A and Kaplan I: Pediatric ameloblastic fibro-odontosarcoma of the mandible: A challenge of diagnosis and treatment. J Oral Maxillofac Surg 71: e45-e57, 2013.

6. Gatz SA, Thway K, Mandeville H, Kerawala C, MacVicar D and Chisholm J: Chemotherapy responsiveness in a patient with multiply relapsed ameloblastic fibro-odontosarcoma of the maxilla. Pediatr Blood Cancer 62: 2029-2032, 2015.

7. Mohsenifar Z, Behrad S and Abbas FM: Epithelial dysplasia in ameloblastic fibrosarcoma arising from recurrent ameloblastic fibroma in a 26-year-old iranian man. Am J Case Rep 16: 548-553, 2015.

8. Loya-Solis A, González-Colunga KJ, Pérez-Rodríguez CM, Ramírez-Ochoa NS, Ceceñas-Falcón L and Barboza-Quintana O: Ameloblastic fibrosarcoma of the mandible: A case report and brief review of the literature. Case Rep Pathol 2015: 245026, 2015.
9. Binnington JA and Adkins KF: Ameloblastic odontosarcoma in a bovine mandible. J Pathol 108: 169-172, 1972.

10. Herzog U, Putzke HP, Bienengräber V and Radke C: The ameloblastic fibro-odontoma-an odontogenic mixed tumor progressing into an odontogenic sarcoma. Dtsch Z Mund Kiefer Gesichtschir 15: 90-93, 1991 (In German).

11. Takeda Y,Kuroda M and Suzuki A: Ameloblastic odontosarcoma (ameloblastic fibro-odontosarcoma) in the mandible. Acta Pathol Jpn 40: 832-837, 1990.

12. Huguet P, Castellvi J, Avila M, Alejo M, Autonell F, Basas C and Bescos MS: Ameloblastic fibrosarcoma: Report of a case. Immunohistochemical study and review of the literature. Med Oral 6: 173-179, 2001 (In English, Spanish).

13. Demoor-Goldschmidt C, Minard-Colin V, Cassagneau E, Supiot S, Oberlin O, D'hautuille C and Corradini N: Ameloblastic fibrosarcoma of the mandible: Report of 2 chemosensitive pediatric cases. J Pediatr Hematol Oncol 34: e72-e76, 2012.

14. Pourdanesh F, Mohamadi M, Moshref M and Soltaninia O: Ameloblastic fibrosarcoma of the mandible with distant metastases. J Oral Maxillofac Surg 73: 2067.e1-7, 2015. 